\title{
Design and Comparative Study Among Antennas of GCS for Telemetry Communication System of UAV
}

\author{
Iswandi ${ }^{1}$, Aria Rangga Suryamanggala ${ }^{1}$, Dewanto Wicaksono ${ }^{1}$, Eny Sukani Rahayu ${ }^{1}$
}

\begin{abstract}
Recently, Unmanned Aerial Vehicle (UAV) becomes a popular and interesting technology for researchers and academics because it has high potential to be implemented in various fields. The UAV operation is managed by a Ground Control Station (GCS) on which requires a communication system to send control signals and acquire data collected by sensors on UAV. The longer the UAV's flying distance needs a higher transmission power budget that can be achieved by increasing transmitter power, increasing receiver sensitivity, or increasing antenna gain. However, the design of communications systems on a flying platform is limited by the following constraints. The transmitter, receiver, and antenna must be compact, lightweight, and energy-efficient to save battery on the vehicle. Although the antenna does not directly influence the energy consumption from the battery, an increase in antenna gain usually requires an increase of the antenna dimension that causes higher weight and or reduces the aerodynamics. Therefore, the most efficient way to increase the link budget is by modifying the antenna on the GCS. This paper describes the analysis of antennas commonly used in GCSs for UAV communication links, namely the Yagi-Uda, bi-quad, and double bi-quad antennas. The antenna is designed to work at a frequency of $433.5 \mathrm{MHz}$ following the transceiver device used. The antenna designs are numerically simulated then fabricated for evaluating their performance. The testing is done on the following issues, i.e. the antenna's basic parameters measurement, the ground-to-ground transmission test, and the ground to air transmission test. The test results show that the double bi-quad gives better performance than the other two other testing antennas.
\end{abstract}

Keyword-Antenna, Biquad, Double Biquad, Ground Control Systems, Telemetry Systems, UAV, Yagi-Uda.

\section{INTRODUCTION}

Unmanned Aerial Vehicle (UAV) recently attracts the attention of researchers and academics due to its great potential for implementing in various fields [1]. This small airborne can be equipped with various sensors and equipment by which various aerial monitoring systems can be developed, for example for agriculture [2], remote sensing [3], forest surveillance [4], coastal surveillance [5], and others.

The obstacle, as well as the challenge in the real implementation of UAV, is the flying range and coverage area which is still bounded by power source and communication link. Regarding the power source, there are two types of $\mathrm{UAV}$, which are liquid-fueled vehicles or electric vehicles. Even though it is liquid-fueled, flying vehicles also requires

\footnotetext{
${ }^{1}$ Department of Electrical and Information Engineering, Faculty of Engineering, Universitas Gadjah Mada,, Jln. Grafika No. 2, Kampus UGM, Yogyakarta, 55281, INDONESIA (Tel. +62-274552305,email: iswandi@ugm.ac.id)
}

electrical energy from batteries to feed electronic devices contained in vehicles, such as controllers, sensors, and communications. The need for electrical energy in this battery creates limits on the flying duration and coverage, moreover for UAV powered by electric motors. Besides, the flight range is also limited by the telecommunications system used to control the vehicle and transmit sensor data [6]. A UAV always has at least a Ground Control Station (GCS). Even though it can fly independently (autonomous), the communication is always needed to transmit data from onboard sensors to GCS or commonly named as telemetry systems.

The communications system consists of a transmitter (Tx), receiver (Rx), and antenna, which is installed on the GCS and UAV to conduct duplex connections. A power budget is calculated to keep the communication of the vehicle and the control station always connected. The transmitter, receiver, and antenna used as well as losses or attenuation of wave propagation in the air influences the power budget. In general, the farther the range from the UAV, the greater the power budget needed, which means a higher power budget is required to improve the flying range. The power budget can be expanded by increasing the transmitting power, the receiving sensitivity, or the antenna gain. Increasing the transmitting power can be done on the GCS side, but it cannot be done on the UAV side because it increases the electric power consumption of the battery. Therefore, increasing the transmitter power is only effective for transmission from the control station to the vehicle, but not vice versa. The increasing receiver sensitivity can be done on both the GCS and UAV sites, because in general it is not related to power consumption. However, receiver sensitivity requires electronic modification of the receiver system, which is usually complicated and relatively difficult. Then, the effective method for increasing the power budget is by increasing the antenna gain.

Either the uplink transmission (from the UAV to GCS) or downlink transmission requires a pair of antennas that function as transmitter and receiver antennas. Since an increase of antenna gain usually requires a larger antenna dimension, an increase in antenna gain is more flexible for antennas that are on the side of the GCS.

Various types of antenna have been investigated for GCS on the UAV communication systems, such as Yagi-Uda [7], [8], bi-quad [9], double bi-quad [10], etc. Since those antennas is designed, fabricated, and tested by different parties, thus the antenna testing environment varies in one to each other. In this paper, research on antenna performance at a GCS is examined by comparing the three antennas commonly used for this purpose. Three antennas are Yagi-Uda, bi-quad, and 

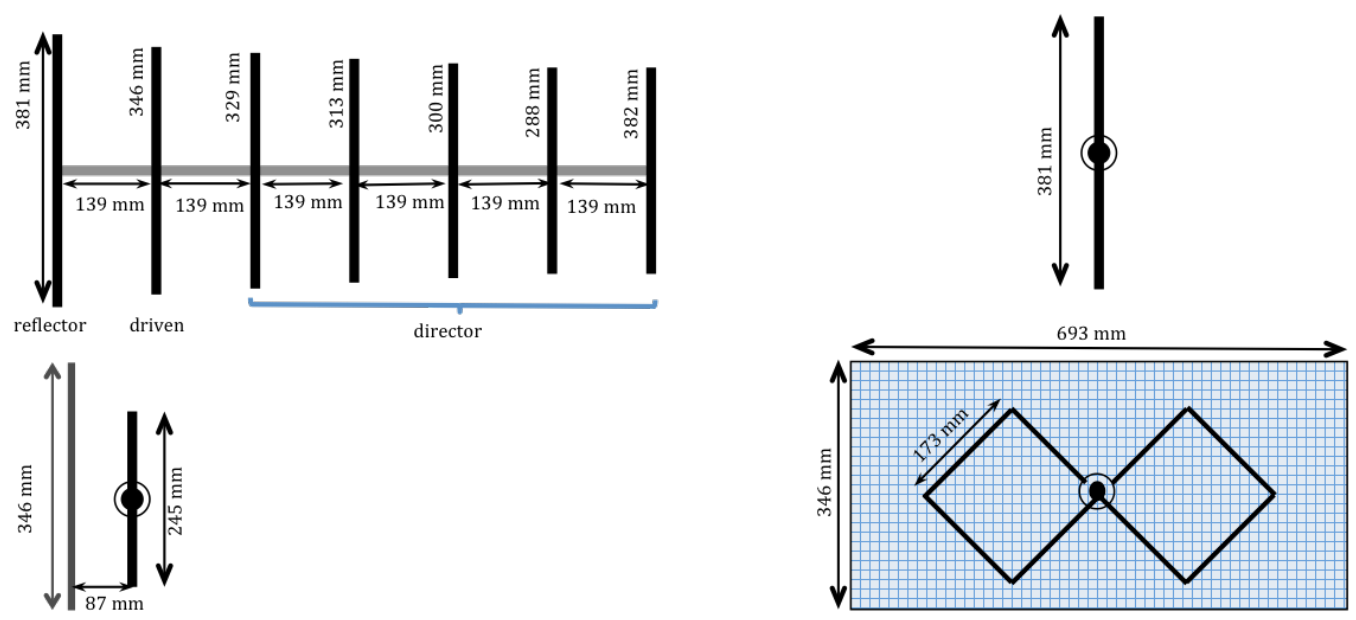

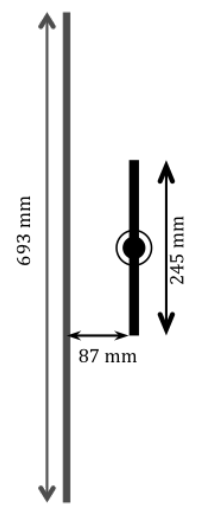

Side view

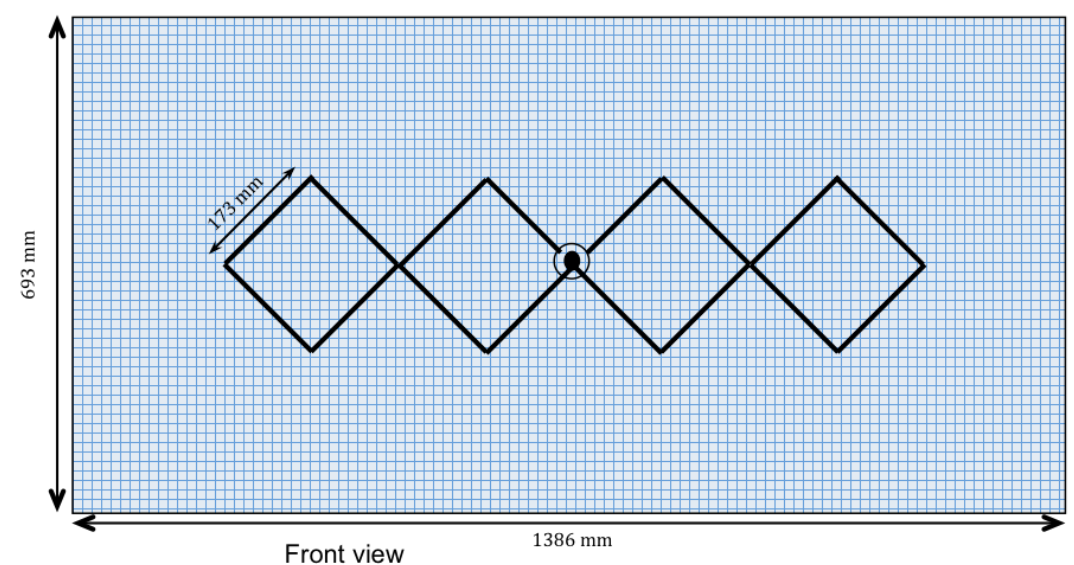

Fig. 1 Structure and dimension of antennas. double bi-quad antennas. The study began with the process of designing an antenna for a working frequency of $433 \mathrm{MHz}$ following the transmitter-receiver device used, then testing the characteristics and performance of the antenna. The tests carried out include testing antenna characteristics, ground transmission testing, and air transmission testing.

\section{ANTENNA DESIGNS}

This study aims to empirically examine the performance of various types of GCS antenna for controlling UAV. For this purpose, we design and fabricate three antenna types, i.e. Yagi-Uda, bi-quad, and double bi-quad antennas, which are commonly used as GCS's antenna in the telemetry communication systems for UAV. Fig. 1 shows the structure and dimension of each antenna.

In the design, the working frequency is adopted from a commercial product of radio transceiver used in hardware development, that is 3DR radio telemetry kits $433 \mathrm{MHz}$, as shown in Fig. 2 [11]. The following sub-section describes the detail design of each aforementioned antenna by setting on the working frequency of $433 \mathrm{MHz}$.

\section{A. Yagi-Uda Antenna}

The Yagi-Uda antenna is a parasitic array antenna consisting of a driven and two types of parasitic elements perform as reflector and director [12]. This antenna has end-

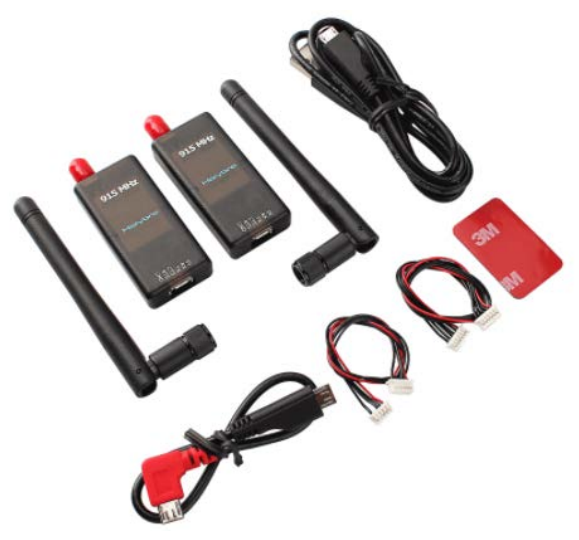

Fig. 2 Radio telemetry kits $433 \mathrm{MHz}$ [11].

fire radiation pattern that means maximum directivity is directed to the array end. The Yagi-Uda antenna is most popular antenna due to its simple structure to design and fabrication.

Regarding the implementation for GCS antenna, the antenna directivity can be improved by increasing the number of elements, but too many elements cause the dimensions to become larger and require a larger antenna rotator for tracking. It should be noticed that using the directive antenna for GCS requires a tracking system to maintain the connection to mobile UAV. 


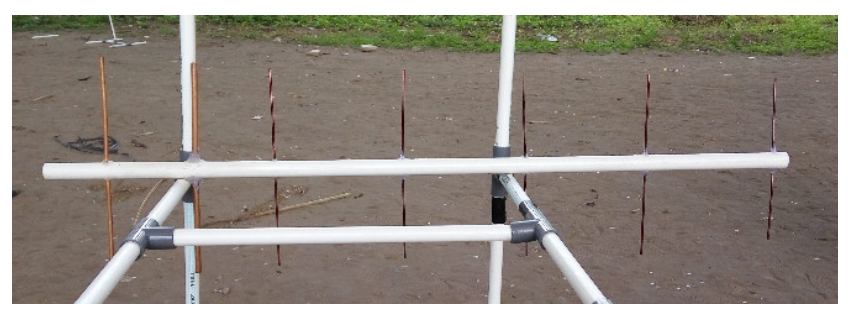

(a)

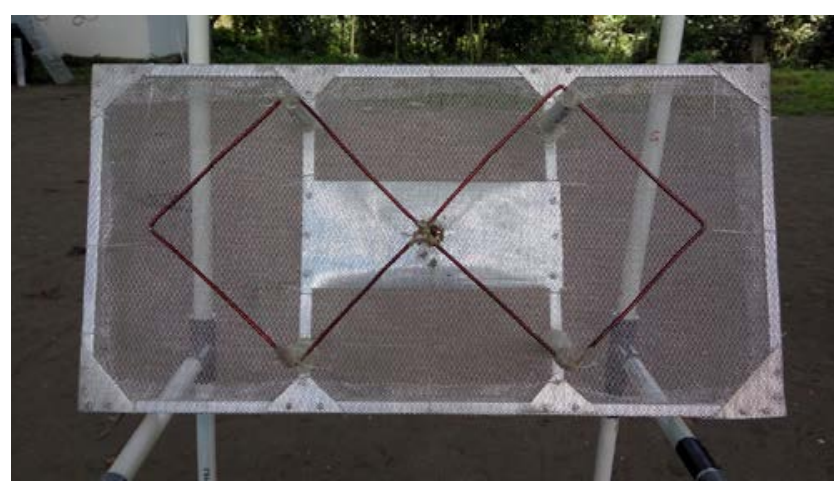

(b)

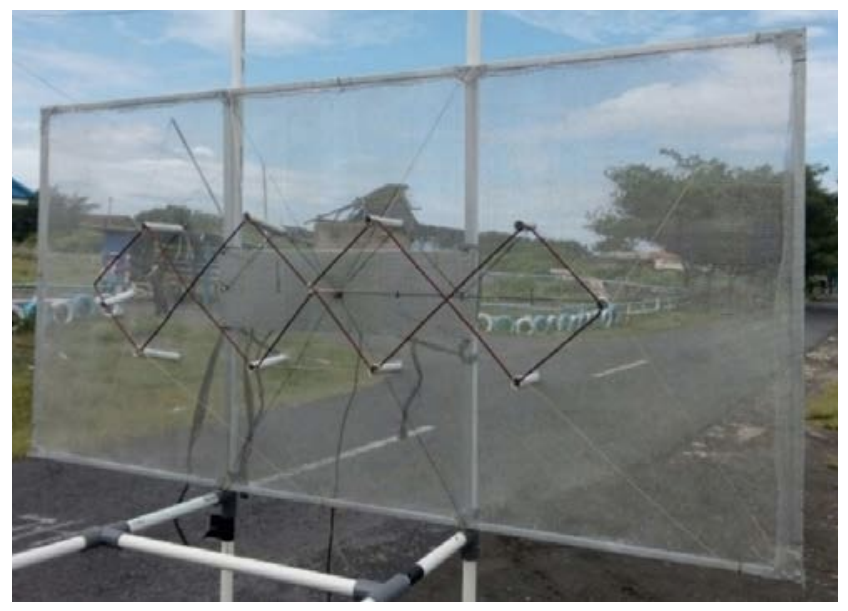

(c)

Fig. 3 Fabrication of antennas, (a) Yagi-Uda, (b) bi-quad, (c) double bi-quad.

The antenna design process is initialized by using theoretical dimensions as listed in [13]. Then, a numerical simulation is conducted to optimize the dimension of antenna element. Considering the effectiveness of number of array elements and limitation of antenna dimension for mounting at antenna rotator, the current works use a 7-elements of array consists of reflector, driven, and five director elements, as figured in Fig. 1.

The antenna is fabricated by using copper hollow pipes with radius of $3.25 \mathrm{~mm}$ and $4.5 \mathrm{~mm}$ for driven element and reflector, respectively, and a $1.65 \mathrm{~mm}$ radius of copper solid wire for directors. Those elements are arranged and fixed on a Polyvynil Chloride (PVC) pipe as antenna boom. The fabricated antenna is shown in Fig. 3(a). It is worth to notice that Yagi-Uda antenna has end-fire radiation pattern where the antenna main beam is at longwise direction.

\section{B. Bi-quad Antenna}

The bi-quad antenna is a modified dipole antenna where the wire is formed in square or diamond shape. The square has four equal sides with length about quarter wavelength. This antenna is simple and easy to fabricate but has high gain and good reliability. It is commonly used in the VHF and UHF applications. Fig. 1 shows the structure and dimension of the designated bi-quad antenna.

The antenna for GCS is required to be directive and pose high gain. Since the bi-quad antenna has omnidirectional pattern, a reflector is added to antenna structure. The reflector has length $\lambda$ and width $\lambda / 2$ and placed behind the bi-quad.

The antenna is fabricated by using a copper hollow pipe with $3.25 \mathrm{~mm}$ radius as bi-quad radiator and an aluminum grill as reflector as depicted in Fig. 3(b). The aluminum grill is chosen to reduce the antenna weight and wind load. This antenna has broadside radiation pattern or crossway main beam direction. This structure gives better balance than YagiUda when it is installed on the rotator.

\section{Double Bi-quad Antenna}

The last antenna is the double bi-quad antenna. This antenna is the extent of bi-quad antenna form by doubling the bi-quad antenna structure. The structure, then, consists of four squares with a quarter wavelength side length. By similar reasons, a reflector also added on antenna structure to form directional pattern. The structure and dimension of each antenna part is shown in Fig. 1.

In the fabrication, the antenna radiator is realized by using $3.25 \mathrm{~mm}$ radius of copper hollow pipe and an aluminum grill for reflector. Fig. 3(c) shows the fabricated antenna. Since the antenna radiator is bigger than bi-quad antenna, it requires wider reflector.

It should be noticed that the polarization of Yagi-Uda antenna is along the element direction, whilst the bi-quad and double bi-quad antennas are perpendicular to the longwise direction. Fig. 3(c) shows the double bi-quad antenna mounted for vertical polarization.

\section{MEASUREMENT CAMPAIGN}

To assess the performance of the designed antenna, a series of measurement campaign was carried out. The measurements consist of two parts, namely the antenna characteristics testing and the transmission testing. Transmission testing consists of two types of ground transmission testing and flight-testing.

\section{A. Antenna Characteristic Test}

Antenna characteristic test aims to measure the basic characteristic parameters of antenna, i.e. return loss, resonant frequency, and radiation pattern. Firstly, the measurement is conducted by connecting the antenna into a Vector Network Analyzer (VNA) in the reflection mode to acquire the reflection coefficient and resonant frequency. Secondly, the antenna radiation pattern is observed by connecting the VNA to the Antenna Under Test (AUT) and a standard omnidirectional dipole antenna in transmission mode. The monopole is set as transmitter while the AUT as receiver. The 


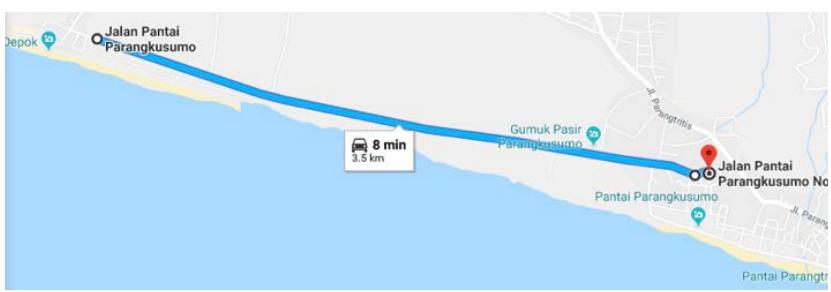

(a)

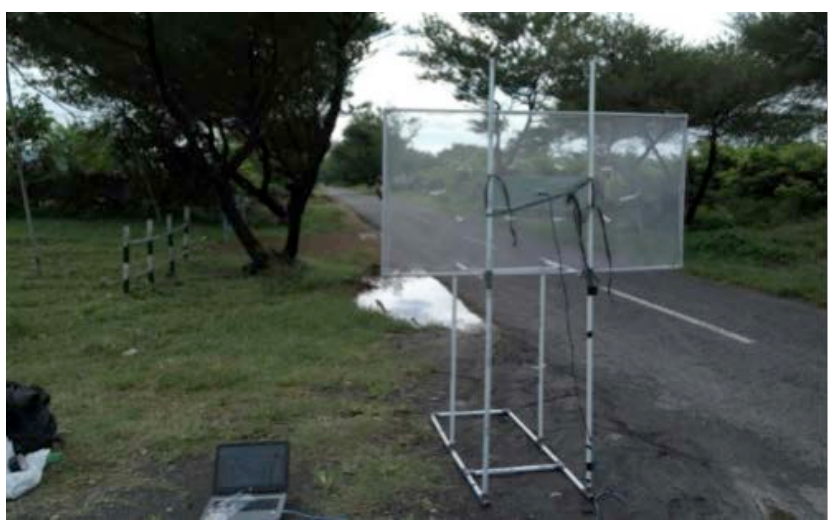

(b)

Fig. 4 Location for test of ground-to-ground transmission, (a) map of location, (b) measurement environments.

AUT is mounted on the rotator by which the pattern on the azimuth plane is measured.

\section{B. Transmission Tests}

The previous measurement concerns the performance of the antenna in the condition of independence to the UAV to which the communication is linked. To consider the real implementation, the measurement is conducted by mounting the antenna on GCS and UAV for transmitter and receiver, respectively. This transmission measurement examines the antenna performance to develop communication from GCS to UAV. The test is realized by two kinds of measurement, i.e. ground to ground and ground to air communications.

In the measurement of ground-to-ground communications, the radio interconnection is observed by moving forward the UAV from the GCS along the ground line. It is done to get the maximum transmission distance because the conditions on land are easier to control than when the vehicle is in flight conditions. The $433 \mathrm{MHz}$ radio telemetry kits, as figured in Fig. 2, are set as transmitter and receiver. As a transmitter, the radio unit is capable of sending signals with 0.5 watts of power, and it has a sensitivity of $-117 \mathrm{dBm}$ as a receiver. The units at the control station use the antenna alternately from the three antennas discussed earlier, while the unit on the UAV uses a monopole antenna.

Regarding the real implementation, the measurement is conducted with the GCS antenna is set to transmit the electromagnetic waves in vertical polarization. To enable the maximum distance of transmission, the measurement campaign is conducted in the road near the beach on which the reflection from environment can be minimized. Fig. 4 shows the map and situational view of measurement location.

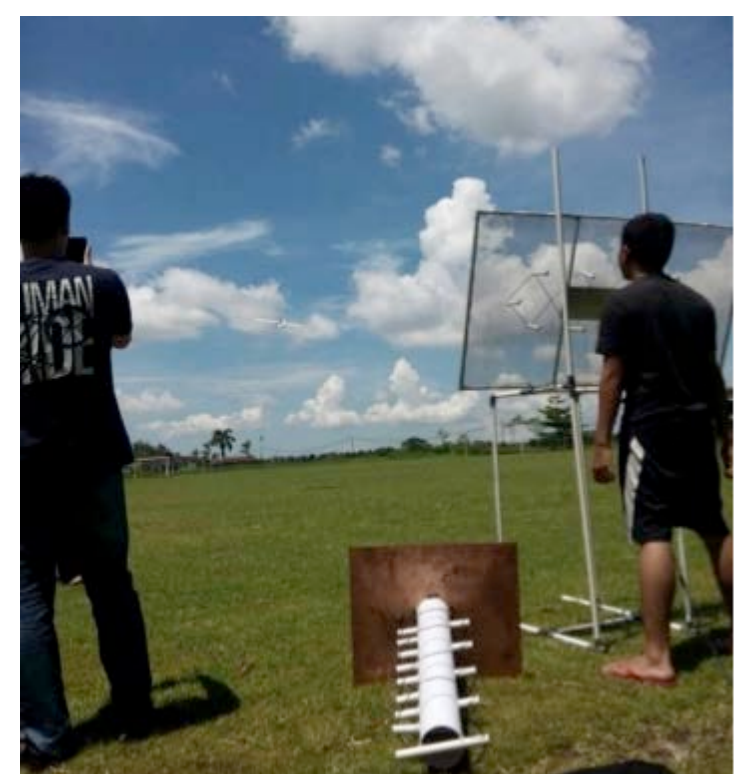

Fig. 5 Location for test of ground to air communications.

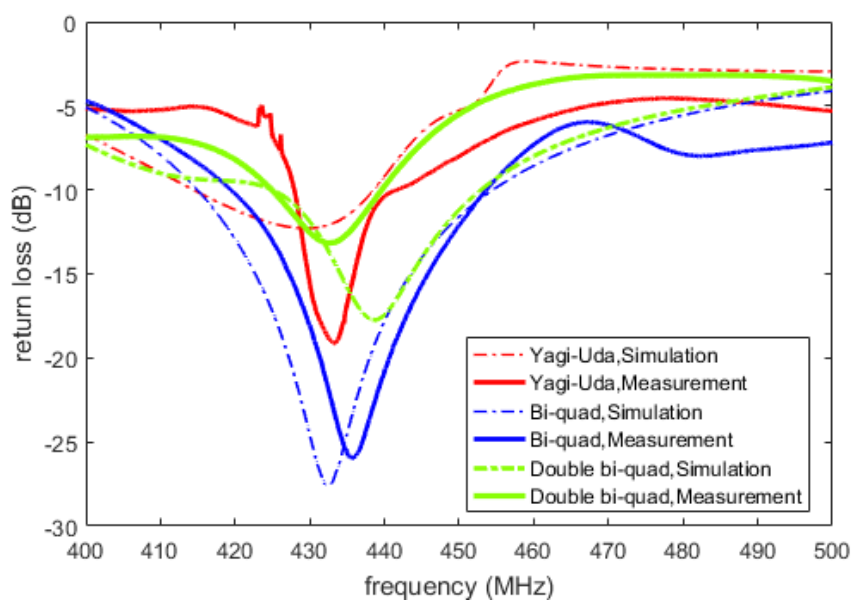

Fig. 6 The simulated and measured return losses of antennas under test.

Next, the ground to air communication test is carried out to look at the maximum range of radio connections from GCS to UAV in the flight mode. The test is conducted by the on-board transmitter which sends message to receiver on GCS. The receiver, then, measures the signal strength of received signal with Received Signal Strength Indicator (RSSI) and compute the error of the received data. The measurement is conducted in open field that has enough space to fly UAV. The location and environment of ground-to-air measurement can be seen in Fig. 5.

\section{RESUlts AND Discussions}

This section discusses the result of measurement campaign conducted to assess and compare the performance of three aforementioned antennas for GCS antenna.

\section{A. Antenna Characteristic Test}

The basic antenna parameters, such as return loss, resonant frequency, fractional bandwidth, and radiation pattern, are acquired by VNA in the measurement. 
TABLE I

The Resonant Frequency AND BANDWIDTH of ANTENNA Under Test.

\begin{tabular}{|c|l|c|c|c|}
\hline \multirow{2}{*}{ Parameters } & \multicolumn{3}{c|}{ Antennas Under Test } \\
\cline { 3 - 5 } \multicolumn{2}{|c|}{} & $\begin{array}{c}\text { Yagi- } \\
\text { Uda }\end{array}$ & $\begin{array}{c}\text { Bi- } \\
\text { quad }\end{array}$ & $\begin{array}{c}\text { Double } \\
\text { Bi-quad }\end{array}$ \\
\hline \multirow{2}{*}{$\begin{array}{c}\text { Resonant } \\
\text { frequency (MHz) }\end{array}$} & Simulation & 423 & 432 & 439 \\
\cline { 2 - 5 } & Measurement & 433.2 & 435.7 & 432.6 \\
\hline $\begin{array}{c}\text { Bandwidth } \\
\text { (MHz) }\end{array}$ & Simulation & 24 & 39.6 & 27.9 \\
\cline { 2 - 5 } & Measurement & 13.6 & 34.3 & 14.7 \\
\hline \multirow{2}{*}{$\begin{array}{c}\text { Fractional } \\
\text { bandwidth (\%) }\end{array}$} & Simulation & 5.6 & 9.2 & 6.3 \\
\cline { 2 - 5 } & Measurement & 3 & 7.9 & 2.4 \\
\hline
\end{tabular}

TABLE II

SUMMARY ON ANTENNA RADIATION PATTERNS.

\begin{tabular}{|c|l|c|c|c|}
\hline \multirow{2}{*}{ Parameters } & \multicolumn{3}{c|}{ Antennas Under Test } \\
\cline { 3 - 5 } \multicolumn{2}{|c|}{} & $\begin{array}{c}\text { Yagi- } \\
\text { Uda }\end{array}$ & Bi-quad & $\begin{array}{c}\text { Double } \\
\text { Bi-quad }\end{array}$ \\
\hline \multirow{2}{*}{$\begin{array}{c}\text { HPBW } \\
\text { (degree) }\end{array}$} & Simulation & 25 & 55 & 50 \\
\cline { 2 - 5 } & Measurement & 30 & 65 & 50 \\
\hline \multirow{2}{*}{$\begin{array}{c}\text { FNBW } \\
\text { degree) }\end{array}$} & Simulation & 55 & 180 & 180 \\
\cline { 2 - 5 } & Measurement & 75 & 175 & 110 \\
\hline $\begin{array}{c}\text { Maximum side } \\
\text { lobes (dB) }\end{array}$ & Simulation & -13.5 & -10.0 & -15.8 \\
\cline { 2 - 5 } & Measurement & -6.0 & -12.0 & -19.0 \\
\hline $\begin{array}{c}\text { Front to Back } \\
\text { Ratio (dB) }\end{array}$ & Simulation & 25.6 & 10.3 & 15.8 \\
\cline { 2 - 5 } & Measurement & 20.5 & 11.9 & 21.1 \\
\hline
\end{tabular}

Firstly, the return losses of the Yagi-Uda, bi-quad, and double bi-quad antenna, respectively, are shown in Fig. 6. In each graph, the measured parameter is combined with the simulated result to convince that fabrication is accordance with the numerical simulation. The measured return loss can be used to calculate other antenna parameters, such as the resonant frequency, bandwidth, and fractional bandwidth, as listed in Table I.

As previously mentioned, the antenna is designed to work on frequency of $433 \mathrm{MHz}$ on which the return loss has to be less than $-10 \mathrm{~dB}$ as the design requirement. The Fig. 6 shows the return losses of three antennas under test from measurement and numerical simulation. All antennas have low reflection coefficient on designated working frequency of $433 \mathrm{MHz}$ on both simulation and measurement. The design of bi-quad antenna has lower reflection coefficient and wider bandwidth. However, all antennas are able to fulfil the requirement to have reflection coefficient less than $-10 \mathrm{~dB}$ in the working frequency of $433 \mathrm{MHz}$.

The frequency responses of antenna as summarized in Table I show that the design of bi-quad performs lowest reflection coefficient and wider bandwidth, but further shifting of resonant frequency from its designated frequency of 433 $\mathrm{MHz}$.

The second part of antenna characteristic test is measuring the directional parameter of antenna, i.e. radiation pattern. The measurement results are depicted in Fig. 7. The measurements on the three antennas show the results that correspond to the results of the antenna simulation. The patterns are in $H$-plane or antennas are set to vertical polarization. The results show

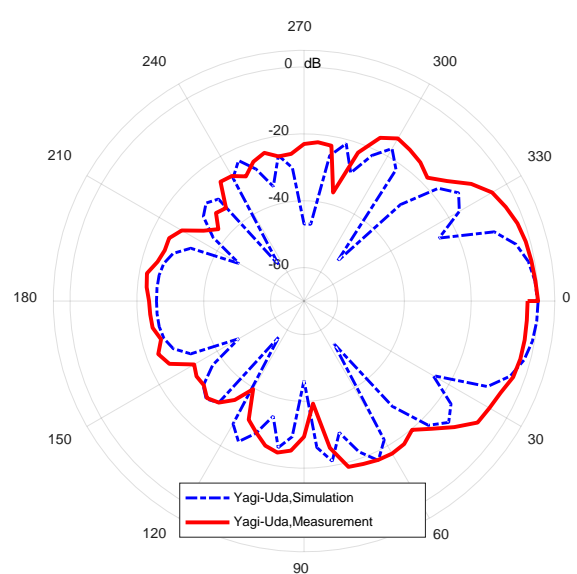

(a)

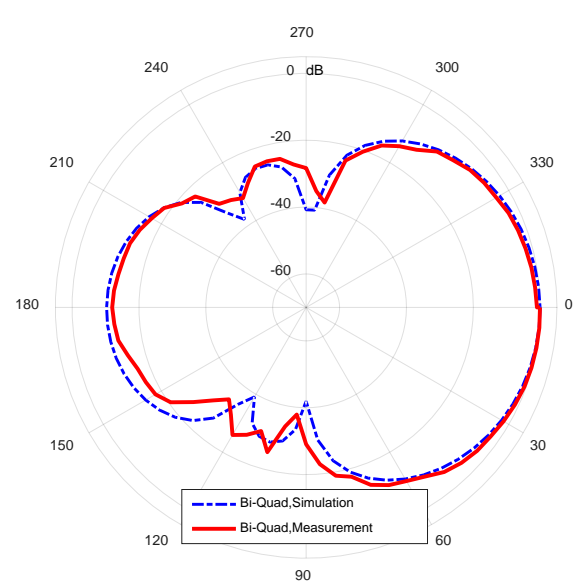

(b)

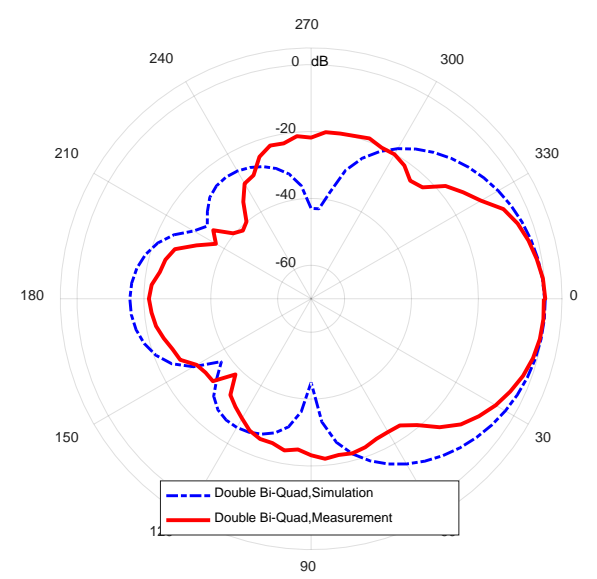

(c)

Fig. 7 The radiation patterns of antennas under test, (a) Yagi-Uda, (b) biquad, (c) double bi-quad.

that all antennas have main lobe in the proper direction and agree w ith the simulation results.

In general, the measurement results agree to the simulation results. Although there are some discrepancies on detail pattern, as shown by parameters as listed in Table II, i.e. Half Power Bandwidth (HPBW), First Null Bandwidth (FNBW), 


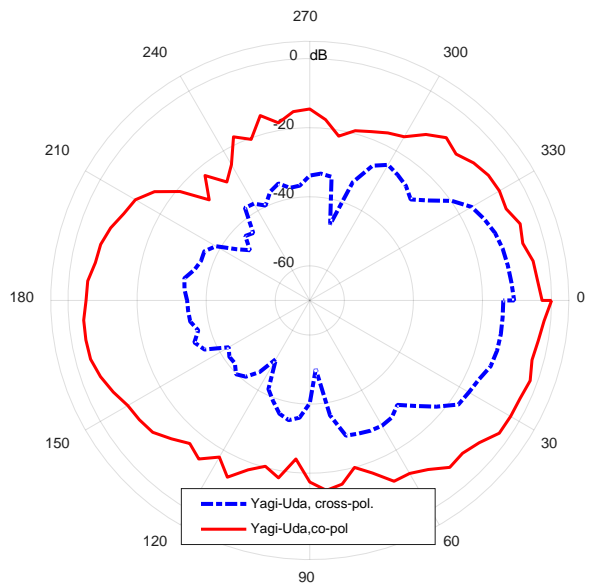

(a)

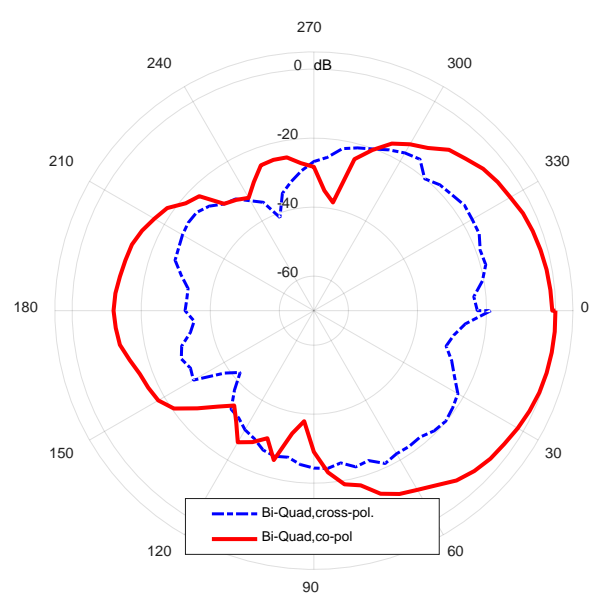

(b)

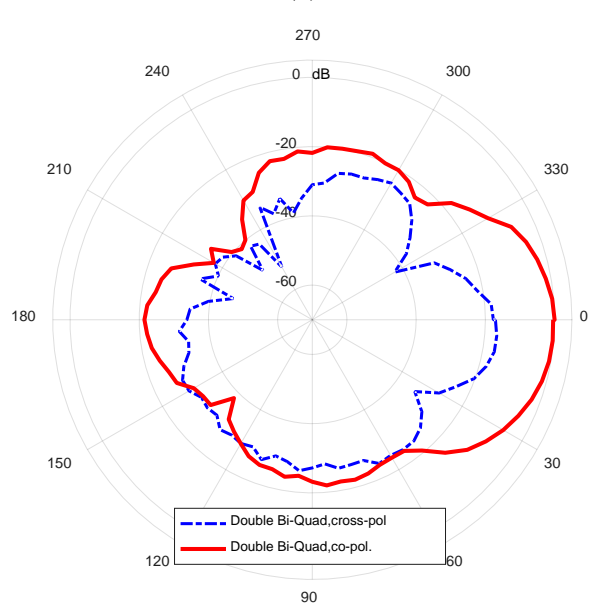

(c)

Fig. 8 The co-polarization and cross-polarization patterns of antenna under tests, (a) Yagi-Uda, (b) bi-quad, (c) double bi-quad.

maximum side lobe, and front to back ratio. These differences are possible to happen because of environmental effect included in the measurements.

Looking at the pattern parameter in Table II, the Yagi-Uda is more directive than other two antennas, since it has lower HPWW, FNBW, and maximum side lobes.

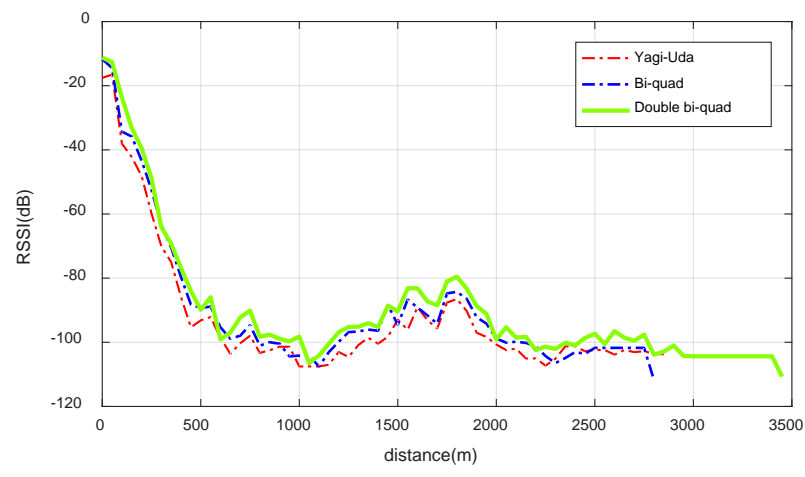

Fig. 9 Result of transmission test for ground-to-ground communications.

The measurements of radiation patterns also acquire the cross polarization patterns. These patterns are observed by considering the dynamic behaviour of UAV antenna. While it flies on the air, the maneuver of UAV can produce misalignment of antenna polarization to the GCS antenna. Thus, the cross-polarized reception possibly gives advantage to maintain the communication of GCS and UAV. The measurement results of cross polarization patterns are depicted in Fig. 8.

\section{B. Transmission Test}

The second test is to assess the performance of antennas to give transmission from UAV to GCS and vice versa. The maximum distance of transmission is measured to evaluate the antenna performance. The test consists of two parts, i.e. the ground-to-ground and ground-to-air transmissions.

The ground-to-ground transmission aims to measure the maximum transmission distance of communication between GCS and UAV. In this scenario, the GCS and UAV are equipped with telemetry radio and antenna. The UAV is held and brought to move away along the straight road by the test personnel.

The measurement results are shown in Fig. 9. The measured parameters are RSSI that can be acquired by telemetry radio on GCS. Although RSSI is relative value of signal strength, but it can be incorporated for performance indicator of transmission. It is worth to notice that the measured RSSI can be obtained if it is higher then the receiver sensitivity. The furthest transmission distance is about 3,433 $\mathrm{m}$, obtained by the double bi-quad antenna as the GCS antenna.

The telemetry radio sends the digital data by breaking it into packets. The quality of transmission can be evaluated by observing the number of packet error transmission. Table III summarized the packet error during ground-to-ground transmission tests. The measurement finding shows that the transmission with double bi-quad antenna performs less packet error rate, about $37.1 \%$. Basically, it just clarifies the results of transmission distance measurements. The packet error commonly occurs when signal closes to the receiver sensitivity threshold. It is happened in almost end of maximum distance.

Lastly, the measurement is conducted to investigate the performance of antenna under test to perform connection 
TABLE III

The PACKet ERRor RATE DURING GROUND-TO-GROUND TRANSMISSION TESTS

\begin{tabular}{|c|c|c|c|}
\hline \multirow{2}{*}{ Description } & \multicolumn{3}{|c|}{ Antenna Under Test } \\
\cline { 2 - 4 } & Yagi-Uda & Bi-quad & $\begin{array}{c}\text { Double Bi- } \\
\text { quad }\end{array}$ \\
\hline $\begin{array}{c}\text { Maximum } \\
\text { distance (km) }\end{array}$ & 2.782 & 2.831 & 3.433 \\
\hline $\begin{array}{c}\text { Transmitted } \\
\text { data (packet) }\end{array}$ & 7,743 & 8,106 & 9,924 \\
\hline $\begin{array}{c}\text { Data with } \\
\text { error (packet) }\end{array}$ & 3,493 & 5,489 & 3,626 \\
\hline $\begin{array}{c}\text { Packet error } \\
\text { rate (\%) }\end{array}$ & 45.1 & 67.1 & 37.1 \\
\hline
\end{tabular}

TABLE IV

COMPARISON OF RSSI AMONG THE ANTENNAS ON TRANSMISSION TESTS

\begin{tabular}{|c|c|c|c|}
\hline \multirow{2}{*}{ Parameters } & \multicolumn{3}{|c|}{ Type of GCS Antenna } \\
\cline { 2 - 4 } & Yagi-Uda & Bi-quad & $\begin{array}{c}\text { Double Bi- } \\
\text { quad }\end{array}$ \\
\hline \multicolumn{4}{|c|}{ Ground-to-ground transmission test (dBm) } \\
\hline 0 & -11.21 & -11.21 & -11.73 \\
\hline 50 & -16.90 & -12.62 & -14.45 \\
\hline 100 & -38.63 & -23.68 & -34.30 \\
\hline 150 & -43.38 & -33.24 & -35.85 \\
\hline 200 & -52.18 & -39.50 & -43.40 \\
\hline \multicolumn{6}{|c|}{ Ground-to-air transmission test (dBm) } \\
\hline 0 & -11.73 & -11.21 & -16.47 \\
\hline 50 & -31.21 & -30.15 & -21.74 \\
\hline 100 & -31.73 & -39.63 & -24.89 \\
\hline 150 & -45.42 & -58.57 & -38.58 \\
\hline 200 & -45.94 & -59.10 & -50.68 \\
\hline
\end{tabular}

between GCS to UAV in flying conditions. The maximum distance of ground to air communication is commonly longer than ground to ground communications. Regarding the power capacity of UAV used for testing is probably not able to reach maximum distance, thus it measures and compares the RSSI for fixed distance, i.e. $200 \mathrm{~m}$.

Table IV describes the comparison of RSSI among the antennas. The results show that the double bi-quad antenna perform statistically higher RSSI for ground to air communications.

The individual characteristic of fabricated double bi-quad is inferior to other, but it can perform farther communication links. Therefore, the optimization on design and fabrication of this antenna is probably able to level up the antenna performance and improve the transmission form or to UAV. This optimization is left for our research future works.

\section{CONCLUSION}

Three types of antenna for the ground control system of UAV communication systems have been successfully designed to operate on $433 \mathrm{MHz}$. The three antennas are Yagi-Uda, bi-quad, and double bi-quad antennas. The antenna performance has been assessed by conducting by a series of measurements, consist of antenna characteristics and transmission tests. The transmission tests consist of groundto-ground communications and ground-to-air communications.

The results show that those antennas have directional patterns, working on designated frequency, and good characteristic parameters (return loss less than $-10 \mathrm{~dB}$ and VSWR less than 2). Furthermore, all designed antennas were successfully tested in the UAV communication system and obtained good results, namely data quality, which was sent almost $100 \%$ at a distance of approximately $200 \mathrm{~m}$. As a comparison, the test recommends that the double bi-quad antenna is superior to two others with a maximum range of $3,433 \mathrm{~m}$ and the packet error rate is $37.1 \%$.

\section{REFERENCES}

[1] H. Shakhatreh, A.H. Sawalmeh, A. Al-Fuqaha, Z. Dou, E. Almaita, I Khalil, N.S. Othman, A. Khreishah, and M. Guizani, "Unmanned Aerial Vehicles (UAVs): A Survey on Civil Applications and Key Research Challenges,” IEEE Access, Vol. 7, pp. 48572-48634, 2019.

[2] J. Kim, S. Kim, C. Ju, and H. Il Son, "Unmanned Aerial Vehicles in Agriculture: A Review of Perspective of Platform, Control, and Applications,” IEEE Access, Vol. 7, pp. 105100-105115, 2019.

[3] G. Pajares, "Overview and Current Status of Remote Sensing Applications Based on Unmanned Aerial Vehicles (UAVs)," Photogramm. Eng. Remote Sensing, Vol. 81, No. 4, pp. 281-329, 2015.

[4] F.A. Hossain, Y. Zhang, and C. Yuan, "A Survey on Forest Fire Monitoring Using Unmanned Aerial Vehicles,” The 3rd International Symposium on Autonomous Systems (ISAS), 2019, pp. 484-489.

[5] I.L. Turner, M.D. Harley, and C.D. Drummond, "UAVs for Coastal Surveying,” Coast. Eng., Vol. 114, pp. 19-24, 2016.

[6] B. Li, Y. Jiang, J. Sun, L. Cai, and C. Y. Wen, "Development and Testing of a Two-UAV Communication Relay System," Sensors (Switzerland), Vol. 16, No. 10, pp. 1-21, 2016.

[7] M.A. Bramantya and A. Yusabih, "Electric Duct Fan UAV with Telemetry System Using Yagi-Uda Antenna,” International Journal Of Scientific \& Technology Research, Vol. 8, No. 9, pp. 96-100, 2019.

[8] G. Nugroho and D. Dectaviansyah, "Design, Manufacture and Performance Analysis of an Automatic Antenna Tracker for an Unmanned Aerial Vehicle (UAV),” J. Mechatronics, Electr. Power, Veh. Technol., Vol. 9, No. 1, pp. 32-40, 2018.

[9] M. Mahardika, G. Nugroho, and E.Y. Prasetyo, "UAV Long Range Surveillance System Based on BiQuad Antenna for the Ground Control Station," Proc. - 14th IEEE Student Conf. Res. Dev. Adv. Technol. Humanit. SCOReD 2016, 2016, pp. 1-5.

[10] (2019) “Arkbird 11dbi 5.8GHz Double Biquad Long Range Directional FPV Antenna - Soft Cable RP-SMA,” [Online], https://www.flyingtech.co.uk/fpv-camera-gimbals/arkbird-11dbi-58ghzdouble-biquad-long-range-antenna, access date: 9-Sep-2019.

[11] (2013) “3DR Radio V2 Quick Start Guide,” [Online], https://3dr.com/wp-content/uploads/2013/10/3DR-Radio-V2-doc1.pdf, access date: 9-Sep-2019.

[12] C.A. Balanis, Antenna Theory: Analysis and Design, 3rd ed., Hoboken, USA: John Wiley \& Sons, Inc., 2005.

[13] W.L. Stutzman and G.A. Thiele, Antenna Theory and Design, Hoboken, USA: John Wiley \& Sons, Inc., 2012. 